\begin{tabular}{|l|c|}
\hline MAGNA & Case Report \\
MEDIKA & Berkala Ilmiah Kedokteran dan Kesehatan \\
\cline { 2 - 3 } & Journal Page: https://jurnal.unimus.ac.id/index.php/APKKM
\end{tabular}

\title{
Optic Neuritis in Pediatric Patients
}

Atik Rahmawati ${ }^{1}$, Dina Fatmawati ${ }^{2}$

17) Opthalmology Department, Faculty of Medicine, Universitas Islam Sultan Agung

\begin{tabular}{l}
\hline \multicolumn{1}{c}{ Article Info } \\
\hline Article history: \\
Received 04 December 2020 \\
Revised 24 December 2020 \\
Accepted 01 January 2021 \\
Available online 01 August 2021 \\
\hline Keywords: \\
Optic neuritis, pediatric, visus \\
\hline Correspondence: \\
atik_rahmawati@yahoo.com \\
\hline
\end{tabular}

How to cite this article:

Rahmawati A, Fatmawati D. Optic Neuritis in Pediatric Patients. MAGNA MEDIKA Berk Ilm Kedokt dan Kesehat. 2021;8(2):102-108

\begin{abstract}
Optic neuritis denotes an inflammation of the optic nerve characterized by loss of vision progressing over a few hours to a few days. Optic neuritis can be classified based on the site involved or its clinical features that is typical and atypical. In some cases, pediatric patients with optic neuritis have atypical symptoms and signs and no comprehensive medical history leading to a challenging diagnosis. In this case series, we report cases of optic neuritis in pediatric patients at different ages and clinical features treated with methylprednisolone $1 \mathrm{mg} / \mathrm{kg}$ body weight and respective management. With a poor initial vision at presentation, the patients recovered rapidly. The diagnosis of optic neuritis was based on anamnesis, ophthalmologic examination, and simple laboratory and physical examination. The administration of steroids at a dose of 1 $\mathrm{mg} / \mathrm{kg}$ body weight followed by a taper of dose can improve vision loss.
\end{abstract}




\section{INTRODUCTION}

Optic neuritis $(\mathrm{ON})$ is an inflammation of the optic nerve characterized by vision loss typically develops over hours or days, accompanied by central or altitudinal visual field defects, dyschromatopsia, especially red color, and periorbital pain on eye movement. Relative Afferent Pupillary Defect (RAPD) is likely except in bilateral and symmetric cases $^{1,2,3}$. Based on the location of the optic nerve involved, the optic neuritis can be categorized as 1. retrobulbar neuritis (2/3 cases) with usual optic disc features, 2. papillitis characterized by hyperemia and optic disc edema, 3. perineuritis involving the optic nerve sheath and neuroretinitis with optic disc edema and macular exudate. In addition, based on its clinical features, optic neuritis is categorized as atypical and typical, which is present without other systemic disease manifestations, including isolated clinical syndromes or diseases associated with systemic demyelination such as multiple sclerosis (MS), acute disseminated encephalomyelitis (ADEM), and neuromyelitis optica (NMO or Devic's disease). Optic neuritis is associated with a viral infection, retinal vasculitis, autoimmune diseases, systemic lupus erythema-tosus (SLE), granulomatous disease, and post-vaccination immune responses ${ }^{1,4}$.

Optic neuritis generally affects young adults, especially female ${ }^{1}$. Pediatric optic neuritis is less common than adults optic neuritis (10 percent of the adult rate) with different clinical features and causes. The comprehensive history of the disease and the onset of symptoms are more difficult to obtain in children. It may only be accidentally detected when the child closes one or after the two eyes are involved. Symptoms of periorbital pain or pain on eye movement are also inconsistent ${ }^{2,3}$.
There have been few reports on retrobulbar neuritis cases in Indonesia, especially in children. It is essential to recognize, provide therapy and evaluate the possible causes that will affect the child's vision and future health. In this retrospective case series, the medical records studies of three pediatric ON patients treated at an ophthalmology clinic were reviewed.

\section{CASE PRESENTATION}

This case series included two patients with bilateral $\mathrm{ON}$ and one patient with unilateral $\mathrm{ON}$ accompanied by edema of the optic disc. All three patients were female. Age at $\mathrm{ON}$ diagnosis was 7, 8 , and 9 years. Vision at arrival was inferior with hand movement and close finger counting. However, final visual acuity (VA) recovered by $20 / 20$ at two weeks to 6 weeks of follow-up. The most extended follow-up in this case in the second case was ten weeks.

\section{Case 1}

A nine-year-old girl (D) presented to the eye clinic with the main complaint of a blurred vision in the left that had started one week period accompanied by headaches after experiencing the last common cold-like symptoms. She was not taking any prescription medication. Two days prior to her first visit, her teacher told her parents that the child was writing in large letters and admitted to having blurred vision. Alloanamnesis showed no history of similar pain, trauma, red-eye, or wearing glasses. She had a history of normal spontaneous childbirth at term with average birth weight and normal body and vision development.

Her general state of health was good. She presented with an uncorrected VA of 20/25 in the right eye, while the left eye only recognizes hand movement $(1 / 300)$. The anterior and posterior 
segments of the eyes were within normal limits, but slightly slowed pupillary reflex at the left eye. There were normal intraocular pressure, orthophoria, normal eye movement, and pain on eye movement was denied. Objective refraction with autorefraction were S-1, $50 \mathrm{C}-0.50$ ax 10 in the right eye and S+1.00, C-1.75 ax 163 in the left eye. There was no color blindness in the right eye, and the left eye could not be assessed. The results of routine blood laboratory tests and erytrocyte sedimentation rate (ESR) were within normal limit. The patient was diagnosed with right and left eyes retrobulbar optic neuritis and was given therapy with methylprednisolone $1 \mathrm{mg} / \mathrm{kg}$ body weight

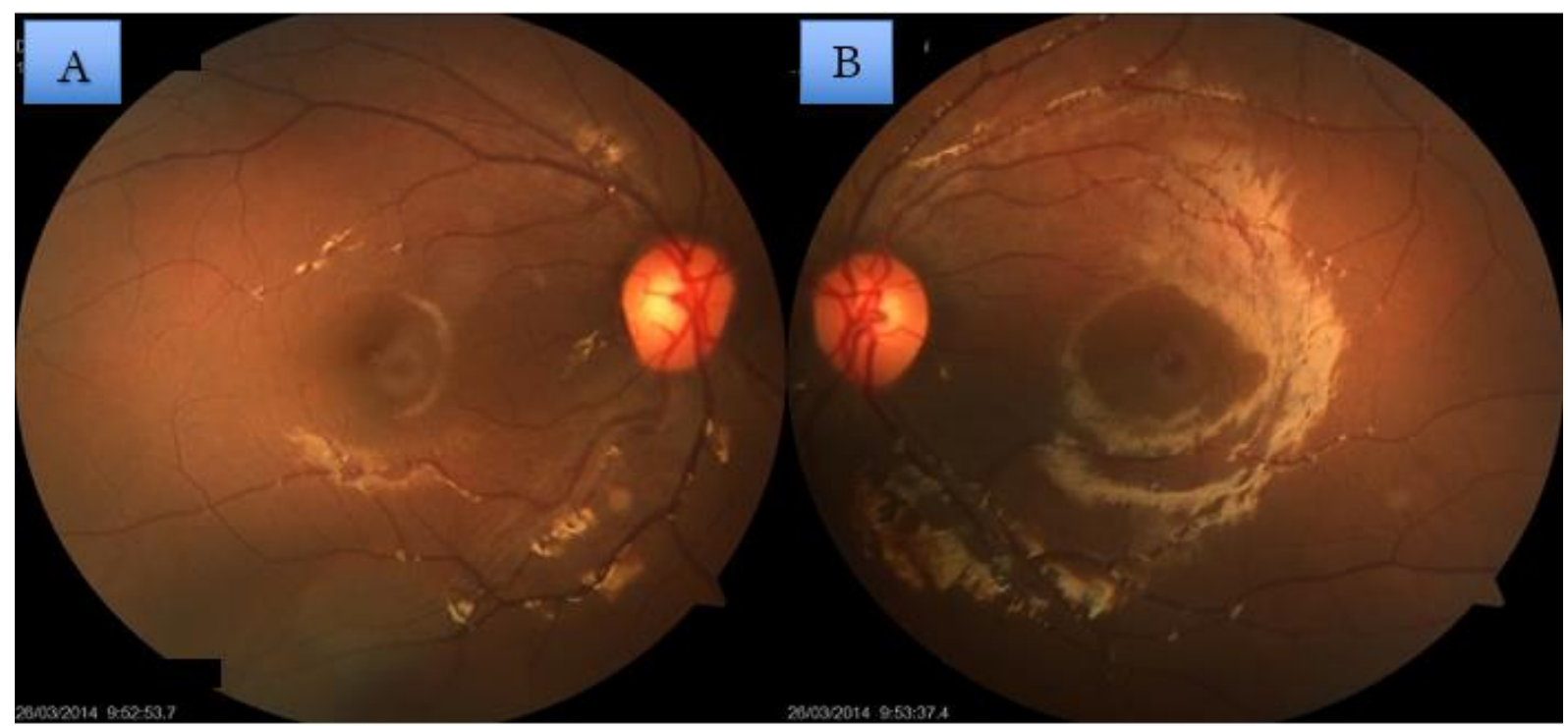

Figure 1. A, B - Colour Fundus Photos - Right and left eyes, respectively, showing posterior segment within normal limit on right (A) and left eyes (B)

\section{Case 2}

An 8-years-old girl (S) presented to the eye clinic with the chief complaint of 8-days blurred vision in the right eye accompanied by a mild headache. She was referred from another ophthalmologist with diagnosis uveitis after 1 week treatment with pills and eye drop without improvement. There was no history of similar sickness, trauma, inflamed eyes, or wearing eyeglasses, according to alloanamnesis. She had a history of normal, spontaneous, term births, with average birth weight and mecobalamin as an adjuvant. The patient was then referred to a pediatrician and was diagnosed with rhinosinusitis and was later given salbutamol and cetirizine therapy.

After three days of steroid therapy, the vision of her right eye improved to 20/20 and left eye to $20 / 60$ pinhole $20 / 30$. Others are within normal limits. She remained on Methylprednisolone 1 $\mathrm{mg} / \mathrm{kg}$ BW for up to 2 weeks, followed by a tapering dose. After four weeks of follow-up, uncorrected visual acuity was 20/20 in her right eye and $20 / 25$ in the left eye. 
eye. There were normal intraocular pressure, orthophoria, and normal eye movement. She negated pain on eye movement. Objective refraction with autorefraction were S- 0.25 C- 0.75 ax 40 on the right eye and S- $0.75, \mathrm{C}-0.25$ ax 163 on the left eye. No color blindness in the left eye and right eye could not be assessed. The results of routine blood tests rate showed mild leukocytosis (12.20 thousand /ul), with mild lymphocytosis $(61.8 \%)$, and an increase in erythrocyte sedimentation rate (ESR) 2(31mm/hour), but immunoserological markers inflammatory C-reactive protein (CRP) was negative. The patient was diagnosed with right eye retrobulbar optic neuritis and was consulted by a pediatrician for associated underlying disease tracking. Based on a routine urine test, the child was diagnosed with a urinary tract infection. She was given methylprednisolone $1 \mathrm{mg} / \mathrm{kg}$ body weight, mecobalamin 1x500 mg, and a pediatrician's management of urinary tract infections.
Follow-up on day 14 after administration of steroid therapy, the right eye vision remained hand movement. Methylprednisolone $1 \mathrm{mg} / \mathrm{kg}$ BW still given for another two weeks and then slowly tapered. At four-week follow-up, there was an improvement on right eye vision to $20 / 60$. Methylprednisolone therapy was continued until the smallest dose, $4 \mathrm{mg}$ per day. At six-week six follow up after administration of steroid therapy, the right eye vision improved to $20 / 25$ with a correction of S + 0.50 to $20 / 20$ and 20/20 on the left eye. Methylprednisolone therapy was discontinued. On final follow-up of 10 weeks, visual acuity right and left eyes were 20/20 without correction, anterior and posterior segments within normal limits. The Ishihara test showed no color blindness. She subjectively feels her right eye was slightly less bright than the left eye.
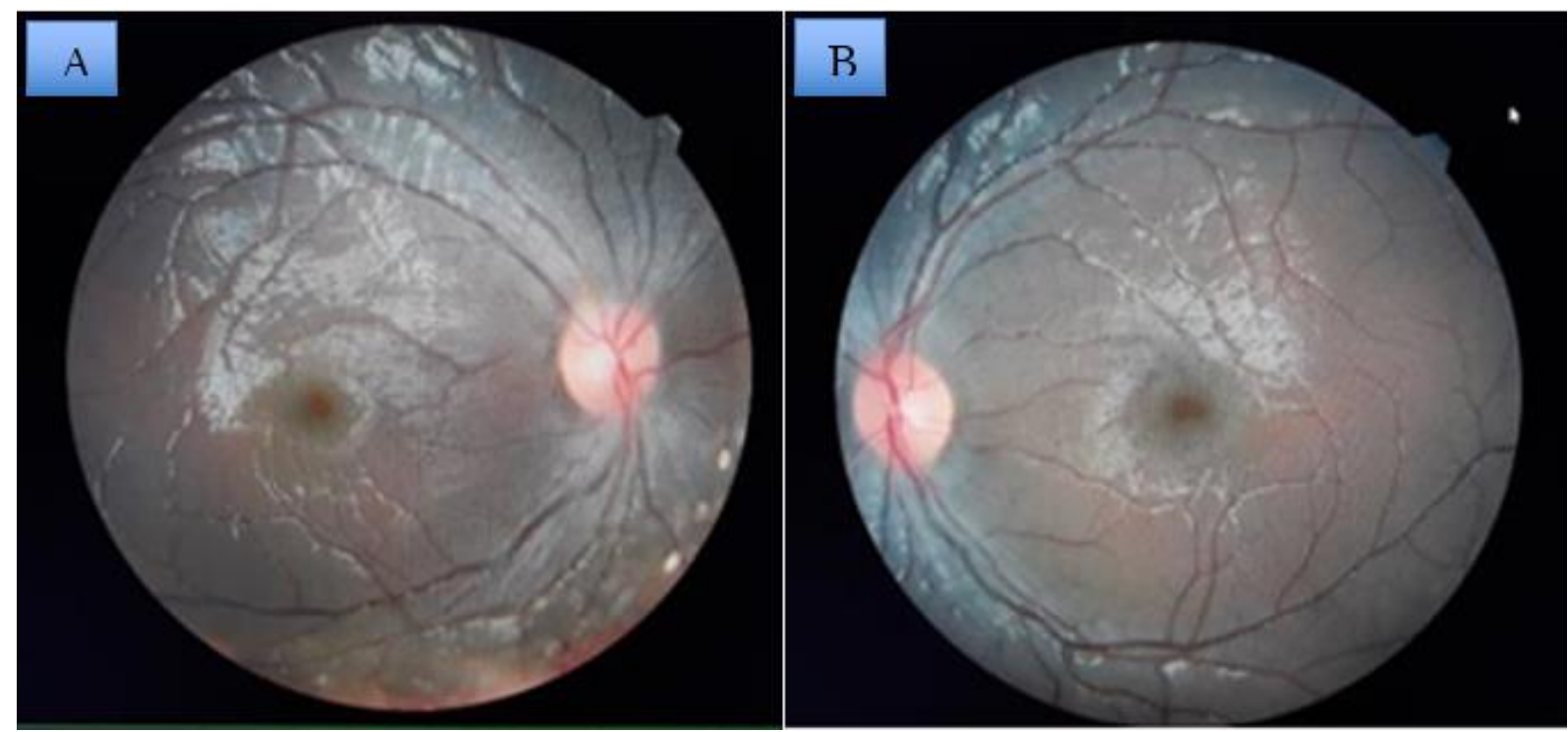

Figure 2. Colour Fundus Photos - Right and left eyes, respectively, showing posterior segment within normal limit on right (A) and left eyes (B)

\section{Case 3}

A 7-year-old girl presented to the eye clinic with the chief complaint of a 2 -days blurred vision in both eyes. She negated red eyes or pain. Alloanamnesis did not obtain a history of previous similar complaints, trauma, or wearing glasses. History of spontaneous birth of normal children at term with average birth weight and normal body and 
vision development. Two months earlier, the child was hospitalized for fever in a different hospital for three days.

The general state of health of the children was good. The visual acuity of both eyes was closed counting fingers. The anterior segments of both eyes are within normal limits, the lens was clear, but slowed pupillary reflexes. The posterior segment shows swollen and hyperemic optic nerve disc at both eyes. There were normal intraocular pressure, orthophoria, and normal eye movement. She negated pain on eye movement. The routine blood laboratory tests showed a slight increase in the number of monocytes $(7.9 \%)$ and erytrocyte sedimentation rate $2(35 \mathrm{~mm} /$ hour $)$. Right and left eyes was diagnosed with papillitis. Methylprednisolone $1 \mathrm{mg} / \mathrm{kg}$ body weight and mecobalamin as an adjuvant. were given to her. For associated underlying disease tracing, the patient was referred to a pediatrician. After1 one week follow-up following administration of steroid therapy, her left eye vision improved to $20 / 120$, but still close finger counting on the right eye. Methylprednisolone 1 $\mathrm{mg} / \mathrm{kg}$ body weight was continued for up to 2 weeks. After the 2-week follow-up, there was an improvement on the right eye vision to $20 / 30$ with a correction of $\mathrm{S}+0,5020 / 25 \mathrm{nbc}$, and $20 / 20$ in left eye vision. Methylprednisolone therapy was continued with a gradually tapered over two weeks. The patient was advised for follow up two weeks later, but the patient did not show up again.
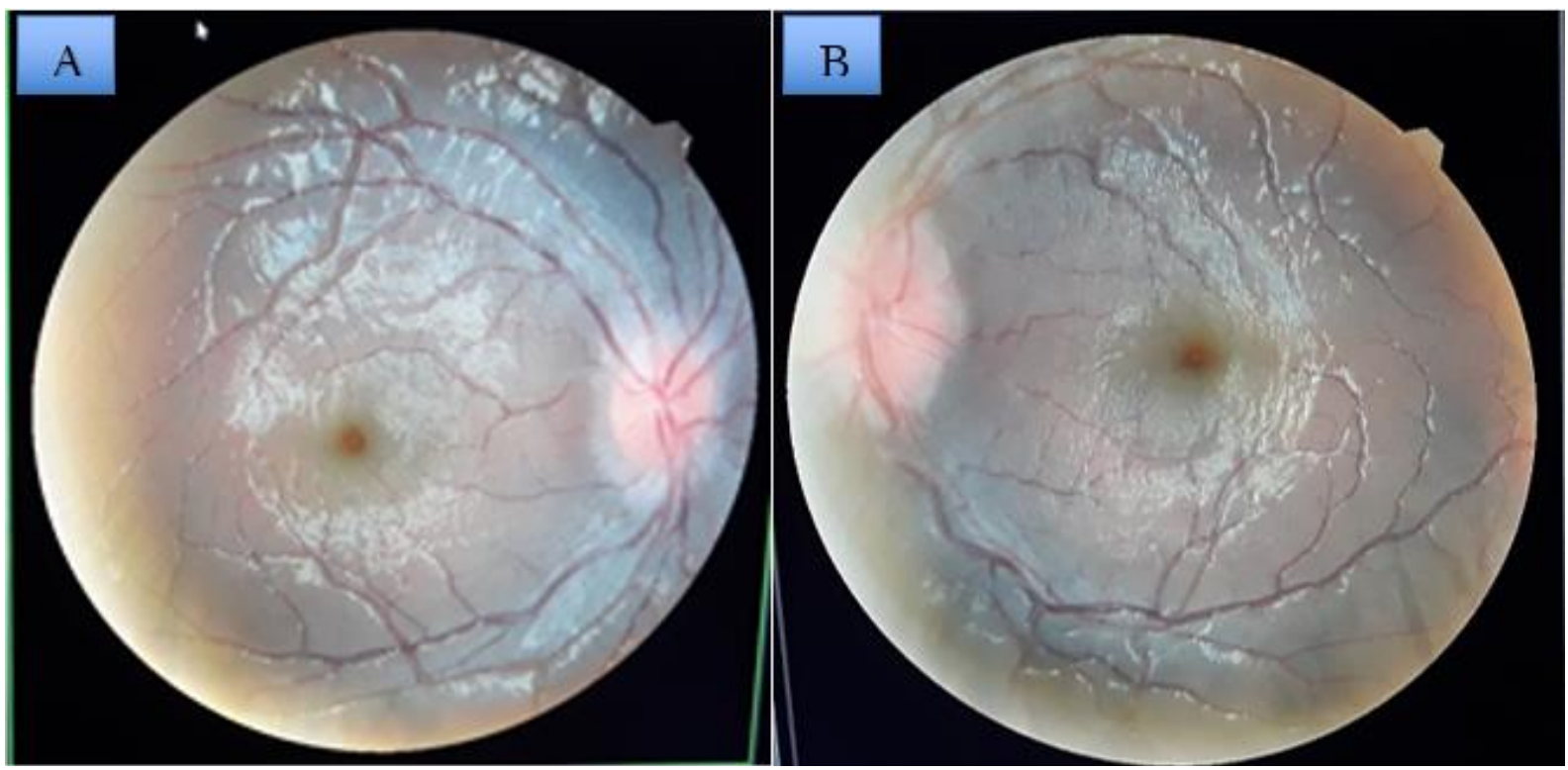

Figure 3. Colour Fundus Photos - Right and left eyes, respectively, showing swollen and hyperemic optic nerve disc on right $(\mathrm{A})$ and left eyes $(\mathrm{B})$.

\section{DISCUSSION}

The diagnosis in all three cases was based on history and ophthalmological examination. Although it is challenging to determine the onset based on the history, a rapid vision deterioration without signs of inflammation in a cooperative children

can support the diagnosis of optic neuritis. The ophthalmological examination included poor and uncorrected visual acuity, which was consistent with no significant refractive error found. Although the pain on eye movement was negated, the patient complained of headaches which were often difficult to determine by children. RAPD is 
an essential clue in unilateral cases, but pupillary abnormalities are difficult to detect in bilateral cases. The retinal funduscopy image of the disc edema supports the diagnosis of papillitis, whereas in retrobulbar cases, the optic nerve and retina may appear normal. The diagnosis of optic neuritis must also exclude other disc abnormalities and inflammatory features in the retina and other parts of the eye. Anamnesis and overall systemic examination are essential to assess the involvement of the nervous and systemic systems that support demyelination syndrome or atypical optic neuritis, which require different treatment.

The clinical features of pediatric optic neuritis differ from those of adult optic neuritis. In pediatric patients, optic neuritis is more familiar with optic disc edema than retrobulbar, and $90-95 \%$ are characterized by severe visual loss of less than $20 / 200$. In children, an excellent visual recovery is more likely with a vision greater than $20 / 40$, whereas visual field disturbances vary and may be challenging to determine in very young children ${ }^{2}$. A meta-analysis on isolated optic neuritis in children aged less than ten years showed that bilateral optic neuritis was more prevalent than unilateral, and vice versa in children over ten years of age ${ }^{5}$. A comparison of the clinical features of optic neuritis from 17 studies among various races around the world also reported by Zhou et al. (2016) showed a high rate of recovery of vision (more than $75 \%$ ) and achieved vision $\geq 20 / 40$ after follow-up ranging from of 1 year to 10 years, and cases of neuritis are more common in girls than boys. In addition, $50 \%$ of cases are bilateral and mostly accompanied by optic disc edema. The study by Zhou on a population of Chinese children also concluded that the patient's age at onset $\leq 10$ years had a significantly better predictive value of vision recovery than older age, and the visual-optic prognosis of neuritis in children was better than in adults. Gender, laterality, initial vision, optic disc edema, MRI images, and therapy did not affect the final visual outcome ${ }^{6}$. Despite the recovery, there may still be subtle changes in their vision. These changes can be in the form of decreased contrast sensitivity, brightness, stereopsis, a field of view, and color vision ${ }^{1}$.

Simple physical and laboratory examinations to identify possible underlying and associated diseases help to diagnose and treat optic neuritis. An underlying cause of optic neuritis is also more common in pediatrics than in adults ${ }^{2}$ Papillitis is more common after viral infection and infectious neuritis than demyelination, although it may also overlap. Children with postviral optic neuritis are generally bilateral and associated with severe visual $\operatorname{loss}^{1}$. A history of flu-like illness may precede the symptoms of blurred eyes. Thus, the diagnosis of optic neuritis is associated with post-infection and vaccination. It is also associated with sarcoidosis, syphilis, tuberculosis, varicella-zoster, EpsteinBarr virus, and therapy with etanercept or inflixi$\mathrm{mab}^{2}$. Viral infection can affect the meninges of the optic nerve or direct parenchyma or is a delayed immune response (postviral optic neuritis) which is generally bilateral with simultaneous onset $^{1}$. Concerning MS, Epstein-Barr virus infection is a commonly associated risk factor, especially with symptoms that appear after childhood ${ }^{7}$.

In typical optic neuritis, improvement is seen within one month. No additional tests are needed to make a diagnosis. Nonetheless, ahead MRI is recommended both to exclude possible intracranial causes causing bilateral disc edema and white matter lesions associated with the risk of ADEM and MS, as well as the decision to use immunomodulating therapy ${ }^{1,2}$. Optic Neuritis Treatment Trial (ONT'T) reported $50 \%$ of adult optic neuritis patients, especially those with lesions on MRI 
develop multiple sclerosis (MS) after 15 years of

follow-up. While in children, based on meta-analysis reported that only 19\% developed MS after 6.3 years of follow-up. Children are at greater risk with increasing age and accompanied with early MRI abnormalities ${ }^{5}$.

The goal of therapy with steroids or immunomodulation is to reduce the number and severity of relapse and prevent further axonal loss and disability. Visual function improvement may occur in $>80 \%$ of untreated patients, stable for several months to 1 year despite possible long-term defects in visual function. The ONTT and several other studies found that the administration of high doses of intravenous corticosteroids effectively improves short-term vision, especially compared to oral steroids and placebo but not in the long term ${ }^{4}$ In adult optic neuritis patients, the ONTT recommends an administration of steroid injection of $250 \mathrm{mg}$ every 6 hours for three days followed by an oral steroid dose of $1 \mathrm{mg} / \mathrm{kgBW}$ per day for the following 11 days. However, there is no standard therapy for pediatric neuritis. Thus, the administration of high doses of steroids for pediatrics remains controversial. Due to the possible loss of vision, intravenous methylprednisolone up to $30 \mathrm{mg} / \mathrm{kg} /$ day, a maximum of $1 \mathrm{~g}$ for 3-5 days followed by oral administration with a gradual taper, is considered ${ }^{2,3}$. Although a study reported no difference in the outcome, the need for steroids for a longer duration of 2 weeks is not established. The study on the efficacy of neuritis therapy in the acute phase other than steroids such as plasma exchange and intravenous immunoglobulin has been still limited and showed contradictory findings ${ }^{3,4}$. The immunomodulation therapy in adult optic neuritis is beneficial in reducing recurrent morbidity and delaying progression from optic neuritis to $\mathrm{MS}^{1}$.
In all three reported cases, a methylprednisolone dose of $1 \mathrm{mg} / \mathrm{kg} \mathrm{BW}$ per day resulted in a rapid recovery of vision. The management of these three cases was outpatient. Therefore, it is crucial to provide education for patients and parents to prevent a decrease in vision due to the Uhtoff's phenomenon, wich is a symptom of decrease in vision triggered by exercise or heat that is common in typical optic neuritis. Therefore, patients are advised to stay at home on hot days and drink cool drinks ${ }^{4}$. Recurrence can be occur in the same or different eyes. In adult optic neuritis, the recurrence rate is $28 \%$ and $35 \%$ at 5 and 10 years of follow-up, respectively ${ }^{4}$. In pediatric patients, 13$36 \%$ of patients with optic neuritis are diagnosed with MS shortly afterward, but recurrency was reported to be only $5 \%{ }^{3}$.

\section{CONCLUSION}

The diagnosis of optic neuritis can be made by anamnesis, ophthalmological examination, and simple investigations in children who are cooperative enough to explain complaints and following examination procedures. A rapid vision deterioration with no significant refractive error and supported by the clinical features commonly found in adult neuritis, can guide a diagnosis of optic neuritis in children. This study suggests that the course of steroids at a dose of $1 \mathrm{mg}$ per $\mathrm{kg}$ body that is tapered and management of the possible underlying risk factor improves visual acuity to achieve the BCVA of 20/25 in 2 to 6 weeks.

\section{REFERENCES}

1. American Academy of Ophthalmology. Basic and Clinical Science Course. NeuroOphthalmology. Section 5(2011-2012). San Fransisco. 
2. Peragallo J. How to Manage Pediatric Optic Neuritis. Review of Ophthalmology. 2018 Apr:1.

3. Yeh EA, Graves JS, Benson LA, Wassmer E, Waldman A. Pediatric Optic Neuritis. Neurology 87 (Suppl2), 2016, August;30:S52-8.

4. Horbakht H, Bagherkashi F. Optic Neuritis, It's Differential Diagnosis and Management. The Open Ophthalmology Journal, 2012, 6, 65-72 65

5. Waldman AT, Stull LB, Galetta SL, Balcer LC, Liu GT. Pediatric Optic Neuritis and Risk of Multiple Sclerosis: Meta-Analysis of Observational Studies. $J$ AAPOS 2011;15:441-6.

6. Zhou H, Wang W, Xu Q, Tan S, Zhao S, Yang M, Peng C, Wei S. Clinical Features and Visual Outcome of Optic Neuritis in Chinese Children. Hindawi Publishing Corporation. Journal of Ophthalmology. Vol 2016, Article ID 9167361, 7 pages

7. Leray E, Moreau T, Fromont A, Edan G. Epidemiology of Multiple Sclerosis. J Neuroophthalmol. 2018 Dec;38(4):462-465. 